\title{
Give Rookies A Chance: A Trust-Based Institutional Online Supplier Recommendation Framework
}

\author{
Han Jiao ${ }^{1}$, Jixue $\mathrm{Liu}^{1}$, Jiuyong $\mathrm{Li}^{1}$, and Chengfei Liu ${ }^{2}$ \\ 1 Data and Web Engineering Lab, \\ School of Computer and Information Science, \\ University of South Australia, \\ SA 5095, Australia \\ \{han.jiao, jixue.liu,jiuyong.li\}@unisa.edu.au \\ 2 Faculty of Information and Communication Technologies, \\ Swinburne University of Technology, \\ Melbourne, VIC3122, Australia \\ cliu@swin.edu.au
}

\begin{abstract}
Trust and reputation systems are widely adopted to help online consumers choose trustworthy suppliers. One problem is that consumers are only attracted by high-reputation holders, which disadvantages the newcomers entering the market, even they provide better goods or services. In this paper, we propose an online supplier selection model in which online consumers interact with a web institution, where online suppliers are registered in, and the web institution will recommend trustworthy suppliers according to a trust-based algorithm. This new recommendation model finds a balance point between minimizing the defective interactions as well as granting opportunities to newcomers. In addition, it saves consumers' efforts to look for trustworthy suppliers. We propose a framework to help realize the new interaction mode. We also use experiments to show its effectiveness.
\end{abstract}

Keywords: trust, trustworthiness, reputation, customer feedback, agent selection, game theory.

\section{Introduction}

Nowadays, using reputation-based methods become the main stream to help determine trustworthiness in the online environment [1. Reputation is derived by scoring and integrating certain attributes of a reputation holder to reflect the global view of the reputation holder. Online consumers give their trust to a supplier by referencing its reputation in relation to the reputations of others. Many reputation-based models using various methods to calculate and compare reputations were studied [2].

Reputation models were improved to better represent the trustworthiness of a supplier. Meanwhile, online suppliers try their best to become high reputation 
holders. All these activities make the whole market towards more trustworthy. However, one serious problem is that online consumers are only attracted by high reputation holders. Newcomers without any historical transactions are hard to be admitted by the market, even though they provide better goods and services. In other words, trust and reputation systems, while providing references to help determine trustworthiness, also place an entry barrier that blocks rookies to enter. This problem is also interpreted to be setting initial reputations for newcomers, since the initial reputation gives online consumers hints to judge trustworthiness. In 1014, it is termed reputation bootstrapping.

Some studies have been conducted on this issues, releasing preliminary results 101112 . In this paper, we solve this problem by designing an institutional recommendation model, between online consumers and suppliers, to grant newcomers some chances without introducing extra risks of potential fraudulence. We first introduce a new concept "reputation utility", based on which we use game theory to construct a model that bridges reputation and trust formation. Then, we propose a framework for a web institution, where online suppliers are registered in, to automatically recommend interactive partners for online consumers. The core selection algorithm is trust-based. We make an implementation of the proposed framework and use experiments to evaluate it.

\section{Related Works}

\subsection{Trust, Trustworthiness and Reputation}

The ubiquity of trust issues offers nothing but more diversified opinions that distract the mainstream understanding of this old concept, since human consciousness came into being. The evolution of trust definition gradually clears the masks on the mystery to result in a notion admitted by the most. Gambetta 4 defined trust as a subject possibility that individual A expects B to perform a given action which A's welfare depends, which is named "reliability trust" in Jøsang et al's survey paper [5]. Appreciation must be given to Mayer et al. for rewarding their seminal work on an integrative trust model in 1995 [3], in which several basic notions related to trust were explicitly distinguished. In that work, trust is defined to be "the willingness of a party to be vulnerable to the actions of another party based on the expectation that the other will perform a particular action important to the trustor, irrespective of the ability to monitor or control that other party". They stressed on vulnerability and regarding trust as willingness to take risk. Definition given by McKnight and Chervany in [6] is of generality, saying that "trust is the extent to which one party is willing to depend on something or somebody in a given situation with a feeling of relative security, even though negative consequence are possible". This is called "Decision trust" in the work of Jøsang et al [5]. Our paper adopts the definition in [6].

Many research works do not explicitly distinguish the concepts of trust and trustworthiness, though they are two related, yet different concepts [3] [7]. Trust is a kind of mental activity in a trustor, while trustworthiness is an attribute 
of a trustee that justifies why a certain amount of trust is worthy to be given to that trustee. Researchers use several dimensions to represent trustworthiness. The most famous classification is proposed in [3] and later adopted in [8, where ability, benevolence and integrity are the three recognized. As the measurement of the extend be to trusted, trustworthiness is like a label stuck on trustees that shines signals to attract attentions. However, a trustor has the "final vote" to decide who would be trusted.

The concept of reputation originates from a contradictory situation where a complete knowledge set on a specific object is unattainable since a full investigation and evaluation is beyond the resource of an individual. It is very natural to consider solving the contradictory situation by relying on aggregated power from multiple individuals. Reputation is the mutual knowledgeable perception that integrates various knowledge sources to reflect a global view of the target. In [3], reputation is defined as "what is generally said or believed about a person's or thing's character or standing", which also conforms to the definitions given in 2] and [8. Basically, reputation is an objective and collective measurement to evaluate a supplier. It is given by the public and is common knowledge held by the whole community. In a situation that one individual has no private knowledge on the other, reputation can be considered as a collective measure representing trustworthiness. This awareness is particularly important in the Web environment, where two online suppliers without any prior interactive experiences is an common case. In this paper, instead of opening the box to check each dimension (ability, benevolence and integrity), we regard reputation as the only indicator of trustworthiness. Further, we use consumer feedback as the base to calculate reputation. This will be discussed in a later section.

\subsection{Trust for Newcomers}

The problem solved in this paper is to promote newcomers in the reputation system without introducing high risks of defective transactions. Currently, there are only a few research focusing on this issue, releasing the outcomes with limitations.

In [1], authors propose that participants with unknown reputation may acquire reputation through the endorsement of other trusted participants and the endorsee's actions directly affect the endorser's credibility. However, it may not be possible for a newcomer to find enough suppliers who are willing to give endorsements, as a newcomer is usually a new competitor to existing suppliers. In [10], Zaki and Athman interpret the trust for newcomers with another term, "reputation bootstrapping", which means to set newcomers a proper initial values. They use both adaptive and assigned approaches to achieve the goal. In the adaptive approach, a reputation community asked their participant to disclose its rate of maliciousness and use an aggregation method (for example, average weighted sum) to calculate an initial reputation for a newcomer. This reputation is adaptive to the latest status of the whole environment since it refers to other existing reputation holders. However, there is no solid evidence showing the average malicious rate is related to any newcomer's initial reputation. In addition, if no enough rate of mali- 
ciousness can be collected, the system can use assigned approach to process, which either asks the newcomer to buy reputation with authenticated credentials or evaluate the newcomers for a while (fake trading to see its performance) and then decide its reputation. Realization of these approaches is difficult, as no one wants to expose their malicious rate; the value of an authenticated credential is hard to measure; and a reputation holder can perform differently during and after an evaluation period. In [12], authors similarity of profiles as the base to determine a newcomer's reputation. They designed models for trust mirroring and trust teleportation to address the issue. But profiles are usually contains privacies and are not easy to obtain and compare in real cases. It may be possible that a newcomer even has no profile since it is very new to the business world. Newcomers can also provide fake profiles to make them look better.

In general, determining newcomer's trustworthiness is still an open issue. In our opinion, this is not a essential problem. The final purpose is that how to get the newcomers involved in without introducing extra risks for defective transactions, which is the problem we will solve.

\section{Reputation Utility and Online Trust Game}

An interaction occurs after a consumer trusts a supplier. Assuming that a consumer has no private knowledge about a supplier, its trust is derived by referencing the supplier's reputation. The purpose that we introduce the concepts of reputation utility and online trust game is to clarify the relationship between reputation and trust formation.

\subsection{Reputation Utility}

Reputation utility $(\mathrm{RU})$ is the benefit that reputation brings to its holder. To better understand it, let us use E-commerce as an example. In a certain market, customers who once bought goods from an E-commerce seller may leave feedbacks. These accumulated feedbacks can be viewed as a kind of reputation by other buyers. It is natural that a seller with nice customer comments has more chances to attract more prospective buyers. In other words, better reputation gains more market shares. This is an evidence that reputation has utilities.

Definition 1. Reputation Utility $(R U)$ is the benefit that a certain reputation value brings to its holder. Given a supplier whose reputation is $r$, we use $\Phi(r)$ to represent the reputation utility of $r$, and name this function as Reputation Utility Function (RUF).

In a reputation-based environment, reputation is changed after certain types of events, such as transactions, customer feedbacks or time durations. The events that can cause a reputation change is termed to be reputation alteration event (RAE). Based on the definition of RU and RAE, we define the concept of marginal reputation utility (MRU). 
Definition 2. Marginal reputation utility (MRU) is the amount of reputation utility change per occurrence of a RAE. It has two components, $\phi_{+}(r)$ and $\phi_{-}(r)$, representing the effects of decrease and increase of $R A E$ on $M R U$. The two functions are called Positive MRU Function and Negative MRU Function.

Positive marginal reputation utility (PMRU) is the reputation utility change per unit for reputation increase. The negative marginal reputation utility (NMRU) is for reputation decrease. MRU is the change amount of reputation utility per combined RAE, which should be distinguished from the change of reputation itself.

Definition 3. Given a certain reputation system $S$, its reputation change policy can be represented by two functions $\eta_{+}\left(r, e_{+}\right)$and $\eta_{-}\left(r, e_{+}\right)$. Given a supplier whose reputation is $r, \eta_{+}\left(r, e_{+}\right)$stands for the reputation change amount for reputation increase given the Event $e_{+}$occurs, and $\eta_{+}\left(r, e_{-}\right)$is the reputation change amount for reputation decrease when Event $e_{-}$occurs. We name two functions as Positive Reputation Change Function and Negative Reputation Change Function, respectively.

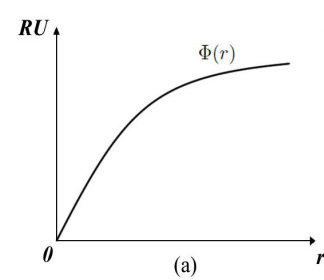

Fig. 1. RU Curve and MRU Curves

The above three concepts are co-related with each other. Their relationship can be represented by the following two equations:

$$
\begin{aligned}
& \phi_{+}(r)=\Phi\left(r+\eta_{+}\right)-\Phi(r) \\
& \phi_{-}(r)=\Phi(r)-\Phi\left(r-\eta_{-}\right)
\end{aligned}
$$

As reputation goes up, the utility of each reputation unit is diminishing. Consider that there is a supplier who has already constructed a good reputation, then the incremental of his reputation score cannot bring him significant utility anymore. This corresponds to the fact that as the reputation goes higher, each reputation unit increase brings less benefit to its holder, which that as two reputation values go higher, the difference of the two reputation holders is not as remarkable as when the reputations are low. This phenomenon in Economics is termed the Law of Diminishing Marginal Utility [13, which makes the reputation utility function as shown in Fig1(a). In addition, reputation is hard to gain but easy to lose. Therefore, $\eta_{+}$should be a decreasing function and $\eta_{-}$is an increasing one, as shown in Fig. 1(b). 


\subsection{Online Trust Game}

Online transactions, being full of remoteness and anonymity, leave participants good possibility to cheat. In a reputation-based circumstance, cheating behaviors usually mean getting an immediate advantage at the expense of lost prospective benefit. The lost prospective benefit is reflected in the change of reputation utility. In our opinion, the interaction between a consumer and a supplier can be modeled by a game, in which each side selects a strategy that maximize their payoffs. This game model describes the gains and losses of each side when selecting different strategies. It creates the linkages between the reputation utility and trust formation. Based on the game theory, we now use a two-player twostage sequential game to describe an online interaction shown in Fig. 2 .

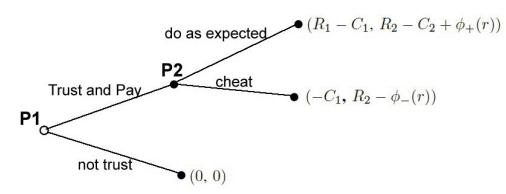

Fig. 2. Online Trust Game

The game has two players: $\mathrm{P} 1$ is the consumer and $\mathrm{P} 2$ is the supplier (reputation holder). At the beginning stage, $\mathrm{P} 1$ decide whether to trust $\mathrm{P} 2$. If not, the game finishes and yields to zero payoffs for both players. Otherwise, the game comes to P2's turn to decide whether to act as expected or to cheat. By acting as expected, P1's requirements are fulfilled. So P1 get benefit $R_{1}$ and pay $\operatorname{cost} C_{1}$. As rewards, $\mathrm{P} 2$ gets the benefit $R_{2}$ plus an increment of reputation utility $\phi_{+}(r)$. But P2 must pay $C_{2}$ as cost. If $\mathrm{P} 2$ cheats, $\mathrm{P} 1$ loses its payment $\left(C_{1}\right)$ and $\mathrm{P} 2$ gets the benefits $R_{2}$ without any cost. As punishment, an extra immediate payoff $\left(\phi_{-}(r)\right)$ due to the cheating behaviors. However, P2's reputation will decrease which results in a decrease of reputation utility.

We assume that the two players both have complete and perfect information. We use backward induction to find the conditions that P1 trusts P2. P1 knows that if the game goes to the second stage, $\mathrm{P} 2$ will choose to act in the way that brings more benefit to P2. Only when $R_{2}-C_{2}+\phi_{+}(r)>R_{2}-\phi_{-}(r)$, P2 chooses to act as expected, which means that only in that case P1 can trust P2. Therefore, the condition that P1 trusts P2 is as follows:

$$
C_{2}<\phi_{+}(r)+\phi_{-}(r)
$$

Because of Formula (1) and (2), the above formula can be refined as follows:

$$
C_{2}<\Phi\left(r+\eta_{+}\right)-\Phi\left(r-\eta_{-}\right)
$$


The above condition tells us that whether P1 trusts P2 depends on numeric relationship between the production cost of the trading goods and the sum of the PMRU and NMRU at the current reputation. In a mature market of a certain products, the cost of each product can be considered as a fixed value (assume that the production technologies are mature and innovation can not be made in a short time). Therefore, the condition for trusting an web supplier is that the sum of PMRU and NMRU of the provider must be greater than a constant value. This intermediate conclusion solve the problem of filtering trustworthy web suppliers.

\section{Trust-Based Auto-selection Framework and Algorithms}

Assume that there are $n$ suppliers in a reputation system, whose reputations are $r_{1}, r_{2}, \ldots, r_{n}$ and who are providing the similar goods or services. We first normalize their reputations. Then we use reputation utility functions to transform reputation values into benefits that reputations bring. After that, we use the trust game model introduced before to filter out trustworthy suppliers. Within all the trustworthy suppliers, we use an auto-selection algorithm to decide a supplier to interact.

\subsection{Normalization}

In a specific reputation system, reputation scores can be scaled into a fix range $[0,1]$. A threshold $r_{o}$ value must be decided to filter out the suppliers who are not considered by consumers. The threshold stands for the psychological baseline of consumers. The suppliers whose reputations are lower than the threshold will never be considered. Given a group of existing reputation scores $r_{1}, r_{2}, \ldots, r_{n}$, the highest of which is $r_{\max }$, for a supplier $r_{i}$, its normalized reputation $R_{i}$ is calculated by the formula that follows:

$$
R_{i}= \begin{cases}\frac{r_{i}-r_{o}}{r_{\max }-r_{o}} & \text { if } r_{i}>r_{o} \\ 0 & \text { if } r_{i} \leq r_{o}\end{cases}
$$

After normalization, primitive reputations $r_{1}, r_{2}, \ldots, r_{n}$ are transformed into $R_{1}$, $R_{2}, \ldots, R_{n}$. If there are $m$ candidate suppliers whose primitive reputation is beyond $r_{o}$, The normalized reputations can be represented as $\left(0,0, \ldots, R_{1}, R_{2}, \ldots R_{m}\right)$, where the number of " 0 " is $m-n$. Truncating the 0 values from the list, we get the final normalized reputations $R=\left(R_{1}, R_{2}, \ldots R_{m}\right)$.

\subsection{Utilization}

Sometimes, even we normalized all the reputations, it may still not be able distinguish reputation holders (as the normalized reputations are still close to 
each other). To solve this problem, we define a Utility Amplification Function (UAF) $\varepsilon(R)$ that is monotone increasing on its definition domain $[0,1]$ and whose second derivative must be greater than 0 . For example, $\varepsilon(R)=R^{n}$ where $n>1$ would satisfy the condition. UAF is optional to use. It helps only when the normalized reputations fail to show clear differences.

Let us now assume that the volume of market demand in a certain duration is $\mathrm{V}$; the average unit cost of interaction for provider is c; the average unit price for consumer is $p$. Our framework defines the Reputation Utility Function (RUF) as follows:

$$
\Phi\left(r_{j}\right)=\frac{\varepsilon\left(R_{j}\right)}{\sum_{i=1, i \neq j}^{n} \varepsilon\left(R_{i}\right)+\varepsilon\left(R_{j}\right)} \times V \times(p-c) \times \rho
$$

Notice that function $f(x)=\frac{x}{S+x}$, where $S$ is a constant value, has the similar monotonicity and concavity as the function shown in Fig. 1 (a). Therefore we use it as the base to form our RUF. For reputation holder $r_{j}, \sum_{i=1, i \neq j}^{n} \varepsilon\left(R_{i}\right)$ is a constant value. In fact, $\frac{\varepsilon\left(R_{j}\right)}{\sum_{i=1}^{n} \varepsilon\left(R_{i}\right)}$ can be viewed as the market share that reputation holder $j$ seizes. Because reputation is not the only factor impacting market share, we use $\rho(0 \leq \rho \leq 1)$ to represent the weight that reputation can impact. They are, then, multiplied by $V$ and $(p-c)$ to get the profit that the reputation $r_{j}$ brings to its holder. By calculating each reputation holder's utility, we finish the second step.

\subsection{Trustworthiness Determination}

Although in the normalization step we have removed some "unacceptable" reputation holders, the rest are just qualified to be candidates. We have to refine the list based on the conditions specified in Formula (4), which is derived from the online trust game model.

We denote Positive and Negative Reputation Change Functions as $\eta_{+}\left(r, e_{+}\right)$ and $\eta_{-}\left(r, e_{-}\right)$. For each reputation holder $j$, its trustworthiness determination logic is as follows:

1. Suppose that in the next interaction it finishes consumer's request as expected, its primitive reputation will be $r_{j}+\eta_{+}\left(r_{j}\right)$. We re-normalize all the reputations to get a new $R^{\prime}=\left(R_{1}, R_{2}, \ldots, R_{m}\right)$. Based on Formula 6. we calculate its reputation utility and denote it as $\Phi_{+}$.

2. Roll back everything to the current status. Then suppose that in the next interaction it cheats, its primitive reputation will be $r_{j}-\eta_{-}\left(r_{j}\right)$. Re-normalize and re-calculate its reputation utility, denoted as $\Phi_{-}$

3. If $c<\Phi_{+}-\Phi_{-}$stands, reputation holder $j$ is trustworthy. Otherwise, mark it as untrustworthy.

4. Roll back everything to current status. 
The above logic can be implemented by the following pseudo code.

Pseudo code for filtering trustworthy suppliers

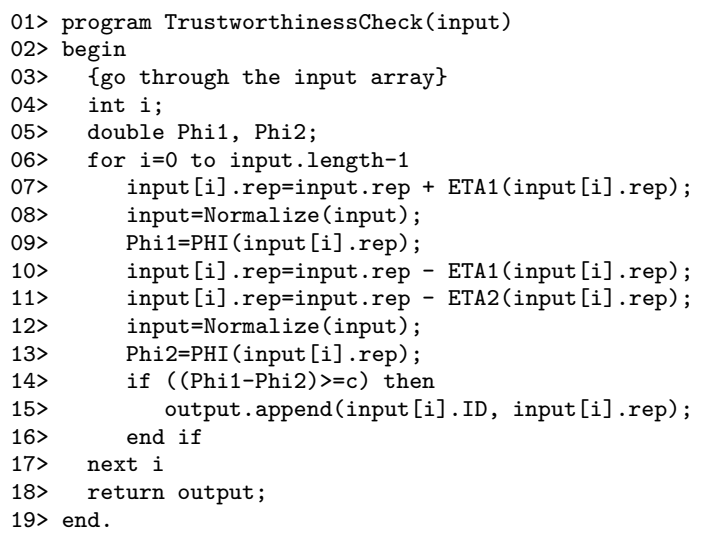

(The input is an array containing IDs and primitive reputations of all the candidate suppliers. The output is an array with IDs and reputations only for trustworthy suppliers. Function ETA1() and ETA2() is the two reputation change functions. Function $\mathrm{PHI}()$ is for calculating the reputation utility. Index of array is from 0.)

\subsection{Recommend Supplier}

Every trustworthy supplier has chance to to be recommended, although their chances are different based on their reputations. We use the following formula to determine its possibility to be selected in a given consumer request:

$$
P_{j}=\frac{\varepsilon\left(R_{j}\right)}{\sum_{i=1}^{n} \varepsilon\left(R_{i}\right)} \times \rho+\frac{1-\rho}{k}
$$

Reputation impacts the market share, as well as other factors, so a weight number $\rho$ is deserved. It is assumed that the impact of other factors together follows a even distribution among trustworthy suppliers. Therefore each supplier deserves $\frac{1}{k}$ where $k$ is the total number of trustworthy suppliers from last step. The following pseudo code implements the logic:

\section{Auto-selection Algorithm}

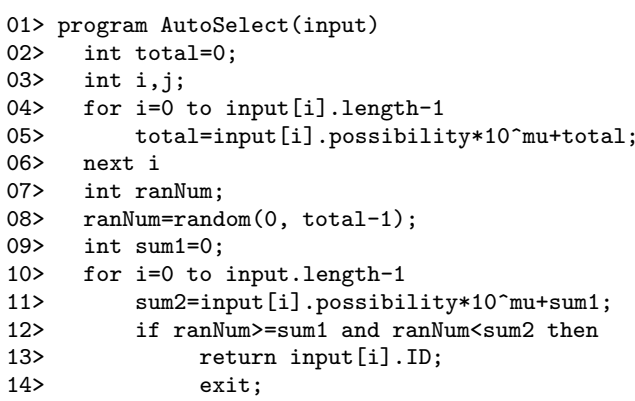




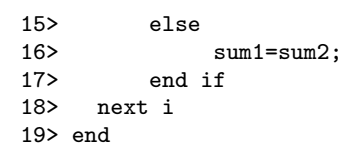

(The input is an array containing IDs and possibility of all the supplier The output is the ID of the selected supplier. The function $\operatorname{round}(a, b)$ returns the value of $a$ with $b$ decimal digits and $m u$ is a parameter of accuracy. The function $\operatorname{random}(x, y)$ returns a random integer from $x$ to $y$. This random number generator follows the even distribution, which means that the chances of the generated number to be any one in the defined range are equal.)

When a supplier quits from the system, all the reputation must be re-normalized so that the market share will be re-allocated. When a new supplier joins, its initial primitive reputation will be equal to that of the lowest trustworthy supplier. This makes it seize a little market share and once it cheats, it falls into the untrustworthy group, which protects the market from defective transactions.

\section{$5 \quad$ Implementation and Experiments}

\subsection{Environment Settings and Implementation}

In an E-commerce market, the total volume is 10000 units. The unit cost is 200 dollars and the unit price is 300 dollars. Positive feedback rate in the last 100 transactions is used to measure reputation. The utilization function is defined as $U=R_{\text {positive }}-4 \times R_{\text {negative }}$, in which $R_{\text {positive }}$ is the positive feedback rate and $R_{\text {negative }}$ is the negative feedback rate. After each transaction, the reputation holder will have $R_{\text {positive }}$ and $R_{\text {negative }}$ and therefore have different utility. We choose $\varepsilon(R)=R^{3}$ as the utility amplification function. The possibility of each trustworthy suppliers to be selected is computed as follows:

$$
P_{j}=\frac{U_{j}^{3}}{\sum_{i=1}^{n} U_{i}^{3}} \times \rho+\frac{1-\rho}{k}
$$

$k$ is the total trustworthy suppliers that has to be decided by going through the four steps described in the last section. $\rho$ is the weight number and later will be given different values in the experiments.

\subsection{Experiment}

Assume that 20 suppliers in the market, whose reputations are randomly generated by a normal distribution with mathematical expectation of 90 and variance of 10, as shown in Table 1.

In each round, there will a consumer coming for only one goods item. After each 100 transactions, one supplier will quit and a newcomer will join. The newcomer's real capability (the ability that they actually gain positive feedbacks from consumers) also follows the a normal distribution with mathematical expectation of 90 and variance of 10 . The way to decide who quits consists of three 
Table 1. Settings for Experiment 1

\begin{tabular}{|l|c|r|r|r|r|r|r|}
\hline Supplier & Reputation & Supplier & Reputation & Supplier & Reputation & Supplier & Reputation \\
\hline Supplier01 & 0.96 & Supplier02 & 0.93 & Supplier03 & 0.94 & Supplier04 & 0.88 \\
Supplier05 & 0.88 & Supplier06 & 0.98 & Supplier07 & 0.74 & Supplier08 & 0.98 \\
Supplier09 & 0.99 & Supplier10 & 0.83 & Supplier11 & 0.78 & Supplier12 & 0.90 \\
Supplier13 & 1.00 & Supplier14 & 0.98 & Supplier15 & 0.89 & Supplier16 & 0.97 \\
Supplier17 & 0.99 & Supplier18 & 0.79 & Supplier19 & 1.00 & Supplier20 & 0.89 \\
\hline
\end{tabular}

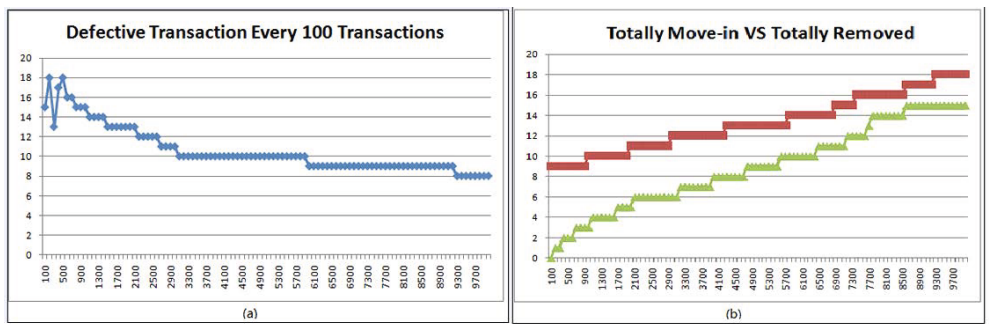

Fig. 3. Online Trust Game

considerations. One is that whether a supplier has been marked as untrustworthy in the system. If so, it will quit. The second consideration is that a supplier with bigger market share should have less possibility to quit because it is doing well. The last consideration is a natural possibility that every supplier may quit for their private reasons. Bearing these in mind, the possibility of supplier $j$ quits is $P_{\text {leave }}^{j}=\rho_{1} \times P_{u t}+\rho_{2} \times P_{\text {nature }}+\left(1-\rho_{1}-\rho 2\right)\left(1-P_{n t}-P_{\text {nature }} * \frac{P_{j}-P \min }{P_{\max }-P \min }\right) . P_{u t}$ is a fixed possibility to quit for untrustworthy suppliers. $P_{\text {nature }}$ is a fixed possibility to quit for supplier's private reasons. $P_{j}$ is the possibility that a supplier $j$ will be selected to interact in each round. $\rho_{1}$ and $\rho_{2}$ are the weights. $P_{\max }$ and $P_{\text {min }}$ are the highest and lowest possibilities of all the suppliers to be selected in a transaction. Fig. 3(a) tells that the number of defective transaction in every 100 transactions goes lower. This shows that the general quality of transactions in the the whole system becomes better. Fig. 3(b) tells the relation between totally moved-in cheaters and total removed cheaters. At the starting point, the system detects several untrustworthy suppliers. As the market moves, they are removed out, but the new untrustworthy cheaters keep on moving in. The two lines converge to each other, which means that the total difference between totally move-in untrustworthy and totally removed untrustworthy suppliers was getting smaller. They finally overlap. Although there are some untrustworthy newcomers moving in, they are removed out quickly as market moves.

\section{Conclusion and Future Work}

Online consumers are always attracted by high reputation holders, which lifts up the market entrance level for newcomers. In this paper, by introducing the concepts of reputation utilities, we use game theories to construct an online trust game, which links the reputation utility with trust formation. We then proposed 
a trust-based auto-selection framework to help consumers select trustworthy online suppliers while balancing the newcomers' involvement. We also make a preliminary implementation of the framework and use an experiment to show its effectiveness.

Our next step is to use real world data to make further examination on the proposed framework. Another possible job is to explore whether there is a general relationship between reputation utility and reputations, which helps to estimate the reputation utility function more precisely.

\section{References}

1. Wang, Y., Vassileva, J.: A Review on Trust and Reputation for Web Service Selection. In: Proceedings of the 27th International Conference on Distributed Computing Systems Workshops (ICDCSW 2007). IEEE Computer Society, Washington, DC (2007)

2. Ruohomaa, S., Kutvonen, L., Koutrouli, E.: Reputation Management Survey. ARES, 103-111 (2007)

3. Roger, C., Mayer, J.H., Davis, F.: n Intergrative Model of Organizational Trust. Academy of Management Review 20(3), 709-734 (1995)

4. Gambetta, D.: Can we trust trust? In: Gambetta, D. (ed.) Trust: Making and Breaking Cooperative Relations, pp. 213-238. Basil Blackwell, Malden (1990)

5. Jøsang, A., Ismail, R., Boyd, C.: A Survey of Trust and Reputation Systems for Online Service Provision. Decision Support Systems 43(2), 618-644 (2007)

6. McKnight, D.H., Chervany, N.L.: The Meanings of Trust, technical Report MISRC Working Paper Series 96-04, University of Minnesota, Management Information Systems Research Center (1996)

7. Gefen, D., Benbasat, I., Pavlou, P.A.: A Research Agenda for Trust in Online Environments. Journal of Management Information Systems 24(4), 275-286 (2008)

8. Gefen, D.: Reflections on the Dimensions of Trust and Trustworthiness Among Online Consumers. ACM SIGMIS Database 33(3) (2002)

9. Artz, D., Gil, Y.: A Survey of Trust in Computer Science and the Semantic Web. Journal of Web Semantics: Science, Services and Agents on the World Wide Web 5(2) (2007)

10. Malik, Z., Bouguettaya, A.: Reputation Bootstrapping for Trust Establishment among Web Services. IEEE Internet Computing 13(1), 40-47 (2009) doi:10.1109/MIC.2009.17

11. Maximilien, E.M., Singh, M.P.: Reputation and Endorsement for Web Services. SIGecom Exchanges 3(1), 24-31 (2002)

12. Skopik, F., Schall, D., Dustdar, S.: Start Trusting Strangers? Bootstrapping and Prediction of Trust. In: Vossen, G., Long, D.D.E., Yu, J.X. (eds.) WISE 2009. LNCS, vol. 5802, pp. 275-289. Springer, Heidelberg (2009)

13. Samuelson, P., Nordhaus, W.: Economics, 19th edn. McGraw-Hill/Irwin (2009)

14. Jiao, H., Jixue Liu, J., Li, J., Liu, C.: A Framework for Reputation Bootstrapping Based On Reputation Utility and Game Theory. In: IEEE TrustCom 2011, pp. 344-351 (2011) 